\title{
Visual-touch perceptual equivalence for shape information in children and adults
}

\author{
CALVIN P. GARBIN \\ University of Nebraska, Lincoln, Nebraska
}

\begin{abstract}
Although there has been substantial developmental research which has compared shape information processing performance under visual and touch conditions, there has been little work that bears on the shape attributes that are routinely employed, or on the similarity between shape attributes employed by adults and those employed by children. The present research was carried out to investigate the visual-touch perceptual equivalence of young children, using multidimensional scaling techniques, and to compare the visual and touch perceptual structures of this age group with those of adults. The results provide evidence for adult-like perceptions of shape among 6-year-olds, in terms of both the patterns of interstimulus similarities and the shape attributes attended to by children using each modality. In addition, it was found that children have somewhat more visual-touch perceptual equivalence than adults do.
\end{abstract}

From the earliest philosophical interests to the most recent physiological efforts, comparisons among the sensory modalities have served as an organizing and unifying theme of perceptual data and theory. There have been three major expressions of this theme. Perceptual equivalence research involves the comparison of the representations of stimuli and/or particular stimulus attributes formed via different modalities (e.g., Marks, 1978). Examinations of intersensory integration have explored the processes and representational results from the combination of information from two or more modalities into a single representation of a stimulus ( $\epsilon . g$., Abravanel, 1981), often one that is richer or more complete than the representation from any single modality (Klatzky, Lederman, \& Reed, 1987). The third approach has involved comparisons of intra- and cross-modal performance on various discrimination and/or recognition tasks. An important topic in the discussion of and research on these issues has been the comparison of visual and touch processing of shape information and the integration of this information under the demands of cross-modal matching and discrimination tasks (e.g., Jones, 1981). This interest in visual-touch comparisons of shape perception may be driven (as it is for me) by the almost unique opportunity to present the same stimulus via two modalities that can potentially process a large number of the same physical attributes of that stimulus.

The present research was designed to assess the visualtouch perceptual equivalence in children's processing of three-dimensional forms, following a procedure that has produced interesting results in studies involving adults (Garbin, 1988; Garbin \& Bernstein, 1984). This proce-

Correspondence should be addressed to Calvin P. Garbin, Department of Psychology, 209 Burnett Hall, University of Nebraska, Lincoln, NE 68588-0308. dure involves the analysis of the holistic shape-similarity judgments of three-dimensional shapes by multidimensional scaling (MDS) techniques that allow the recovery of the attributes upon which the judgments were based. The research was prompted by two related aspects of the literature, both of which are discussed more fully below. First, there has been little use of psychophysical techniques to examine visual-touch perceptual equivalence in children, and virtually no research has been done using three-dimensional stimuli (see Abravanel, 1981, for a discussion of the utility of three-dimensional stimuli for visual-touch comparisons). Second, the major source of research about children's visual and touch shapeinformation processing has consisted of tasks (e.g., crossmodal matching tasks) that involve memory and sensory integration/comparison skills, and so, the resulting data do not directly address perceptual equivalence.

Much attention and empirical research during the last 30 years has addressed the similarities and differences between visual and touch shape-information processing in children and adults. Several of these studies have been cleverly designed to uncover differences in the stimulus sources of the different matching errors made under visual and touch conditions (e.g., Pick, Pick, \& Thomas, 1966). These studies have supplied information about the differences in the types of feature discriminations that can be made using vision and touch. However, these studies have not supplied direct evidence about the shape attributes to which subjects routinely do attend when perceiving, identifying, and/or discriminating between stimuli. Put another way, the attributes that can be used successfully as the basis of a discrimination judgment may not be the same attributes that are routinely employed in the construction of the perceptual representation of the stimuli. The indirect evidence supplied by these data is important; it is unlikely that a poorly discriminated attribute would be an important part of a perceptual representation. MDS anal- 
yses of similarity rating data, such as those employed in the present research, are well suited to the comparison of the stimulus attributes to which subjects attend when perceiving stimuli via vision and touch.

Cross-modal discrimination and matching tasks have also provided an important approach for examinations of visual and touch shape-information processing (for excellent overviews from a developmental perspective, see Abravanel, 1981, and Jones, 1981). In these tasks, subjects first examine a standard shape using one modality and then examine one or more comparison shapes using the same or the other modality and select the standard (or indicate that it is not present, depending on the task). These studies have produced three consistent conclusions. First, both children and adults are able to transfer shape information between the two senses; that is, cross-modal performance is typically above chance levels. Second, cross-modal performance is consistently poorer than visual and often poorer than touch performance. Third, crossmodal performance is poorer among children than among adults. Stated differently, the difference between intraand cross-modal performances is greater among children than among adults.

The performance of cross-modal tasks requires the combination of two perceptual abilities, perceptual equivalence (forming similar visual and touch representations) and sensory integration (accurate combinations or comparisons of these representations), as well as the obvious memory component. One theoretical limitation of research involving cross-modal tasks is that the data do not readily allow separation of the roles of the two perceptual components and thus give no direct information about perceptual equivalence. Rather, this type of research seems to assume visual-touch perceptual equivalence for all stimuli (i.e., that the visual and touch representations of any particular stimulus are the same). This assumption is apparently made with great conviction, for only a few of these articles mention the issues of perceptual equivalence (see Garbin, 1988, for a related discussion). In keeping with this assumption, the usual interpretation is that poorer cross-modal performance is produced by a less-thanperfect sensory integration/comparison capability. An alternate explanation is suggested by a consideration of the role played by perceptual equivalence in cross-modal tasks. If the visual- and touch-based representations of a given stimulus are dissimilar (because of limited or incomplete perceptual equivalence), then poor cross-modal performance with respect to that stimulus would not be surprising.

A similar approach can be taken to the interpretation of the differential cross-modal performances of children and adults. Starting with the assumption of perceptual equivalence in both children and adults, as researchers seem to have done, the usual interpretation of poorer cross-modal performance in children is that they have poorer intersensory integration/comparison than do adults. An alternate explanation is that children have less com- plete perceptual equivalence than adults do, and so they are making more comparisons between "dissimilar stimuli" than adults are, which leads to poorer cross-modal performance.

\section{Examinations and Definitions of Perceptual Equivalence}

Important evidence regarding adults' visual-touch perceptual equivalence has come from psychophysical studies of shape attributes. In these studies, perceptual equivalence has been studied and defined in terms of the correlation (across stimuli) of visual and touch ratings of specific stimulus attributes. For example, Owen and Brown (1970) reported that the correlation between the visual and touch ratings of the complexity of two-dimensional polygons was .98 , indicating nearly complete perceptual equivalence for that attribute (additional studies are discussed in Garbin \& Bernstein, 1984; Marks, 1978).

In a study of adults' perception of holistic shape (Garbin \& Bernstein, 1984), subjects were asked to sort a set of 24 three-dimensional stimuli into groups that were similar in overall shape and to rate each stimulus on a series of shape attributes (e.g., size and complexity), using either vision or touch. Three different "definitions" or indications of visual-touch perceptual equivalence were studied in that research. As in Owen and Brown (1970), visual and touch ratings of specific shape attributes were generally strongly correlated. In addition, the relationships among the shape attributes were similarly perceived via vision and touch, as indicated by very similar three-factor structures (size, shape, and symmetry). MDS techniques were applied to the sorting data to uncover and describe the comparability of shape attributes that acted as the bases for the similarity judgments, another possible indication of perceptual equivalence.

Comparisons of separately derived visual and touch MDS solutions led to three conclusions. Both visual and touch subjects used attributes of size (e.g., height, compactness, etc.) as the major similarity dimension and attributes of shape (e.g., angularity, complexity, etc.) and symmetry as the second similarity dimension. Also, about $67 \%$ of the stimuli had very similar positions in the visual and touch perceptual (MDS) structures, which was interpreted to mean that there was strong perceptual equivalence for these stimuli. However, $33 \%$ of the stimuli $o c-$ cupied substantially different positions in the visual and touch solutions, which was interpreted to mean that these stimuli were perceived with less visual-touch equivalence. The first two findings combine to suggest that the term perceptual equivalence makes a useful general statement about visual-touch shape perception; the third tempers that general statement with the reminder that not all stimuli are perceived in exactly the same way via vision and touch. In particular, the finding suggests that although adults use the same attributes during visual and touch perception, they quantify stimuli somewhat differently on the basis of these attributes via the two modalities, which thus 
results in different positions in the perceptual (MDS) structures. The overall interpretation was that there was partial equivalence between the two senses. This is the term originally invoked by Gibson (e.g., 1966; though it is difficult to know if the term was used in anticipation of the lack of complete equivalence for particular attributes or in recognition that not all attributes of a stimulus are available to pairs of modalities), and it is consistent with the results of the extensive comparisons of vision and touch made by Marks (1978).

The relationship between perceptual equivalence and cross-modal performance was examined in subsequent research (Garbin, 1988). It was determined that one important limitation of previous perceptual equivalence was the absence of a definition or measure of the perceptual equivalence of individual stimuli. Such a measure of perceptual equivalence, called cross-modal dissimilarity (CMD), was derived from a comparison of MDS models of visual and touch perceptual structures from previous work (Garbin \& Bernstein, 1984). The measure for each stimulus was the Euclidean distance between the positions of that stimulus in the visual and touch MDS solutions. The rationale behind the measure was that the position difference of a stimulus in the visual and touch MDS solutions indicates differences in the perceived similarity of that stimulus and others, and so reflects differences in the perception of that individual stimulus (see Garbin, 1988, for a more detailed discussion). The measure is actually of perceptual nonequivalence, because larger values indicate greater positional dissimilarity. The stability and replicability of both the perceptual structures and the CMD values of individual stimuli were shown with two procedures, each of which revealed that 8 (of 24) stimuli consistently had larger CMD values (less perceptual equivalence), but that 16 had small values (greater perceptual equivalence).

Two experiments were then conducted in which the levels of perceptual equivalence of the stimuli were manipulated within both intra- and cross-modal conditions of a shape-memory task. These revealed that although cross-modal and intramodal performance were at the same high level when "equivalent" stimuli were involved, cross-modal performance was substantially poorer than intramodal performance when "less equivalent" stimuli were involved. These results supply important experimental validation of the interpretation that the CMD value for each stimulus reflects its perceptual equivalence. In addition, the importance of separating perceptual and cognitive components of cross-modal performance was highlighted. The results also emphasize that the study of perceptual equivalence must involve comparisons of visual and touch perceptual structures (Isaac, 1970), in addition to examinations of integrative (i.e., cross-modal) processing.

This brief review of research comparing visual and touch processing of shape information suggests the theoretical importance of a direct assessment of similarities and differences in the attributes and attribute quantifications that children employ under visual and touch perceptual conditions. This information will allow a comparison between the perceptual equivalence of adults and that of children, both quantitatively and qualitatively. In addition, the results will add to the information gained from past research about the types of attributes children can use as the basis for discrimination, to provide a more complete picture of children's shape-perception processes and abilities. Finally, information about children's perceptual equivalence will help in the interpretation of the findings that children have poorer cross-modal than intramodal performance and that children's cross-modal performance is poorer than that of adults.

On the basis of the stability of adult perceptual structures for these stimuli when different subsets are used (Garbin, 1988), as well as the stability of MDS solutions across data collection procedures (e.g., Wish, 1976), it was predicted that the adult sample in this study would reproduce the visual and touch MDS solutions and CMD values that had been found in previous research. Specific predictions for the children were more difficult to derive. One possibility was that there would be greater heterogeneity in the attributes attended to among children than there would be among adults, which would make scaling analyses of similarity judgments difficult and suspect. Another prediction might be made on the basis of the finding (discussed above; cf. Jones, 1981) of somewhat larger differences between intra- and cross-modal discrimination performance in children than in adults. It was possible that this larger difference occurred in children because children have less perceptual equivalence than adults do. ${ }^{1}$ Thus, one might expect the children to exhibit larger stimulus-by-stimulus CMD values than the adults do.

\section{METHOD}

\section{Subjects}

Thirty-nine children (74-90 months of age) were recruited from a private school in a large southwestern city. Twenty-one children participated in the touch condition and 18 participated in the visual condition of the similarity judgment task. Ten of these children from each modality condition also participated in the attribute rating task. The data from 1 child from each perceptual condition were excluded from the analysis, because they did not meet the criterion set for the screening task. Sixteen undergraduate psychology students from a university in the same city served as the adult sample; 8 participated in each perceptual condition. Gender was equally balanced in each of the four design conditions of each age group, except that there was 1 fewer female than there were males in the child visual group.

\section{Materials}

The 12 solid three-dimensional stimuli shown in Figure 1 were chosen from a larger set of 24 (Garbin, 1988; Garbin \& Bernstein, 1984). The stimuli were made of air-drying pottery clay, painted black. Each stimulus fit within a $9-\mathrm{cm}$ sphere and rested on a prescribed base throughout the procedures. As described above, previous research had demonstrated and replicated that 8 of the original 24 stimuli were found to be less perceptually equivalent (had 

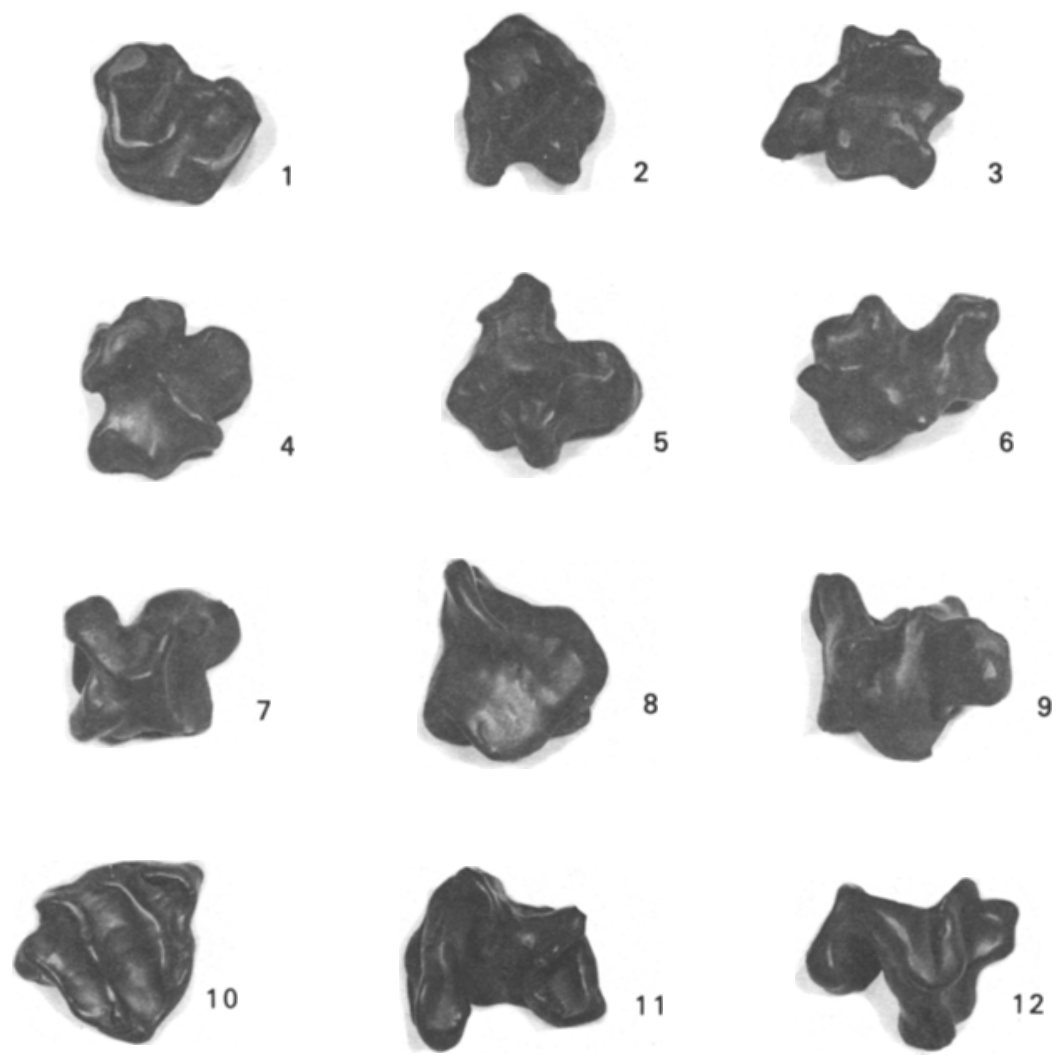

Figure 1. The twelve shape stimuli used in the study.

higher CMD values), whereas the other 16 stimuli were more equivalent (had lower CMD values). The 12 stimuli illustrated in Figure 1 included 6 less equivalent stimuli (numbered 1-6 in Figures 2-3) and 6 more equivalent stimuli (numbered 7-12). An additional set of 6 stimuli was selected for use during the screening/practice trials.

\section{Procedure}

Similarity judgment task. Pilot work revealed that the children could perform neither similarity groupings nor pair-comparison ratings with acceptable reliability. Thus, similarity data were collected using the method of triads (Torgerson, 1958). On each trial, the subjects were presented with three stimuli and asked to indicate which two were most similar in overall shape, and then to indicate which two stimuli were most different. Each of the 220 possible triads were presented twice within each design condition.

The subjects first completed five practice and screening trials using the set of six practice stimuli. Because these stimuli had produced very consistent triadic choices during pilot work with both age groups, the trials could also be used as a screening task to determine whether or not each subject understood the task. Data from 2 subjects who made three or more nondominant similarity and/or difference choices during the last three of the practice trials were not included in the analysis. Following the practice trials, each child completed between 20 and 35 trials. The children were asked if they wanted to continue after every 5 th trial, and the session was ended when they chose. Each adult completed the practice trials and 55 trials.

Attribute rating task. Because of the difficulty in obtaining physical measures of the shape characteristics of these stimuli (except for simple attributes such as vertical height), in previous research (Garbin \& Bernstein, 1984) measures of the stimuli had been ob- tained from subjects' ratings of various attributes (e.g., complexity, symmetry, angularity, size). Pilot work revealed that ratings of this type given by children were very unstable (the best average interrater reliability, .35, was obtained for judgments of height). Thus, an alternative procedure was adopted.

After the MDS solutions from the child's visual and touch conditions were obtained (as described in the Results section below), sets of five stimuli were selected that represented positions along each solution axis. Each set of five stimuli was presented to a child via the same modality that the child used to perform the triads task, and the child was asked to order the stimuli with respect to a particular attribute. For example (see Figure 2A), Stimuli 11, 8, 2, 1 , and 10 might be used to represent the visual size axis, with 11 and 8 having negative weights, 2 a weight near zero, and 1 and 10 having positive weights. Subjects would, in this example, be presented with stimuli and asked to "line them up from the smallest to the largest." Each child completed 16 trials, 4 trials containing different sets of five stimuli for each of the following attributes: (1) small versus large; (2) compact versus dispersed (the expression "spread out" was used with the children); (3) simple versus complex; and (4) rounded versus angular features. ${ }^{2}$ The stimuli for each trial were chosen with the constraint that there be one stimulus from each of five partitions on the relevant axis, larger than $-1.2,-1.2$ to $-.50,-.50$ to $.50, .50$ to 1.2 , and larger than 1.2 .

\section{RESULTS AND DISCUSSION}

\section{MDS Analyses}

The response data from each of the four conditions were transformed into separate $(12 \times 12)$ dissimilarity matrices, and MDS solutions of one through three dimensions were 


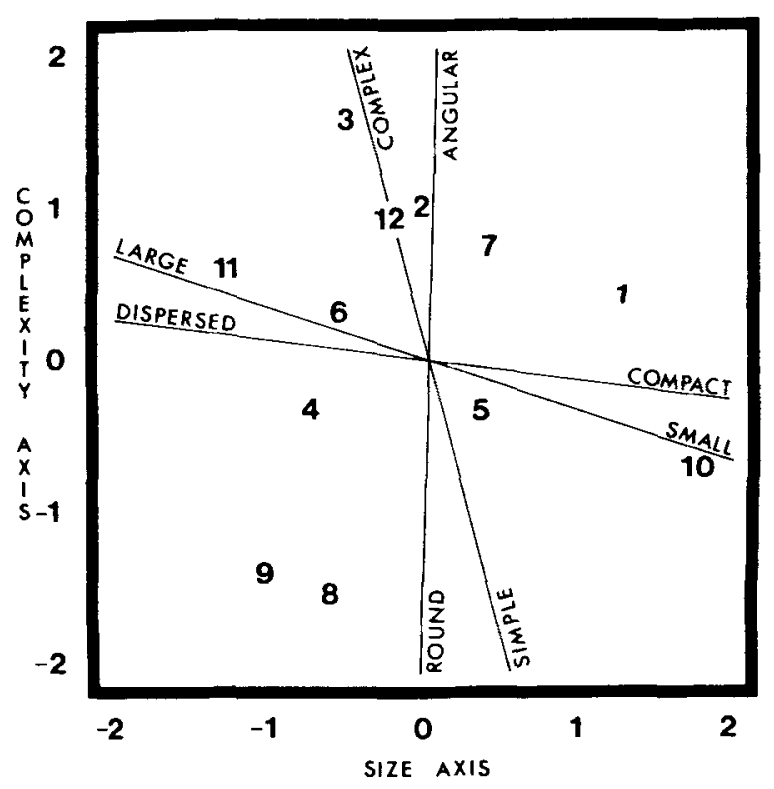

A

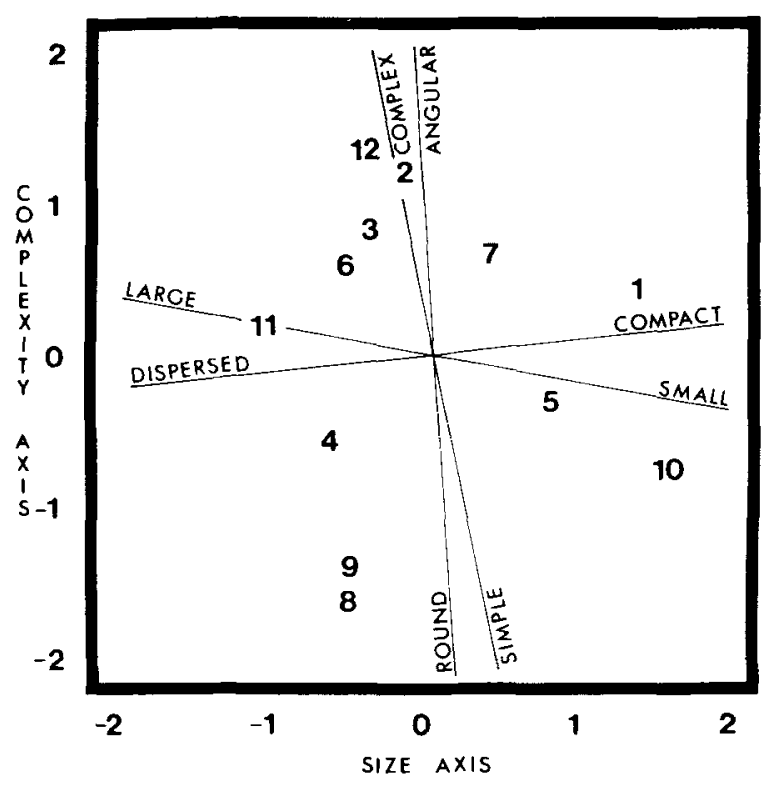

C

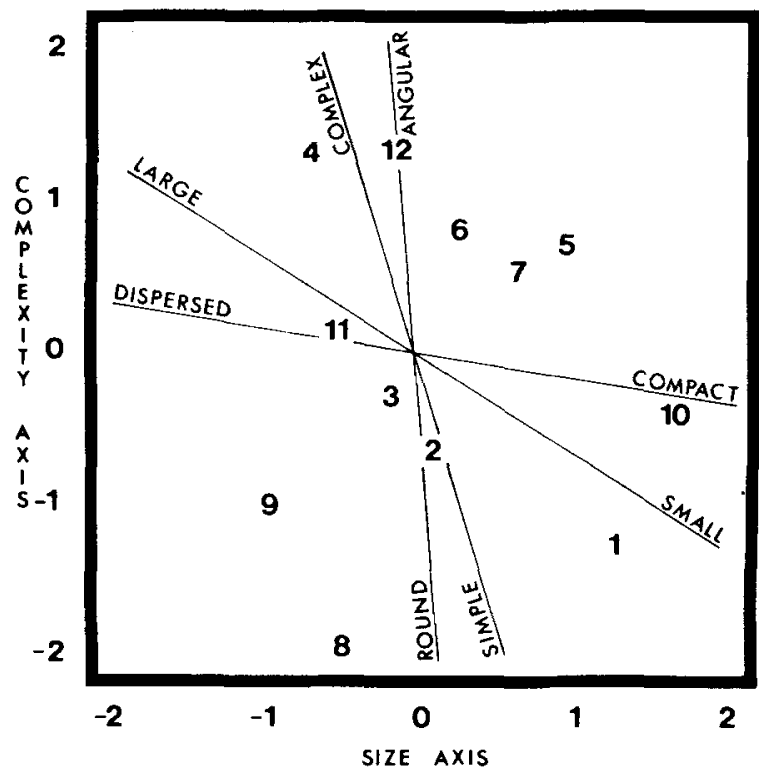

B

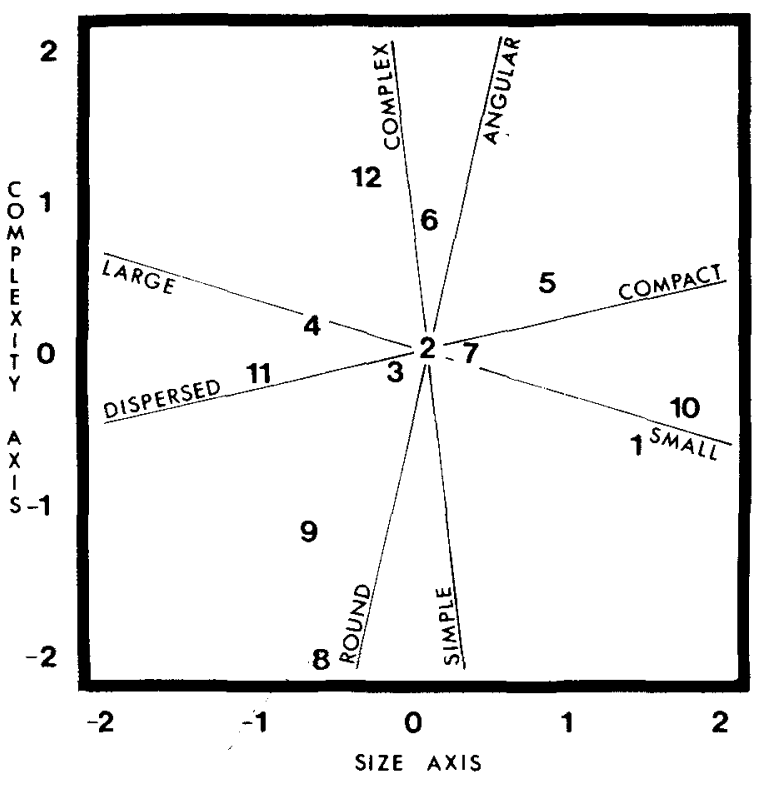

D

Figure 2. Two-dimensional multidimensional scaling solutions from each of the four conditions, after orthonormal rotation to positions of maximum congruence: (A) adult visual; (B) adult touch; (C) child visual; (D) child touch. The positions of the four attribute dimensions in each panel were determined as is described in the text.

obtained for each condition (matrix), using a nonmetric scaling procedure (ALSCAL; Young \& Lewyckyj, 1979). The $R^{2}$ and stress measures used to assess the fit of the MDS models to the data are shown in Table 1 (these values are from solutions for which the algorithm chose the starting configuration, and they are nearly identical with those for which arbitrary initial configurations were imposed). As had been found in previous research with these stimuli (Garbin, 1988; Garbin \& Bernstein, 1984), a two-dimensional solution fit the data from each condition substantially better than did a one-dimensional model, and there were only negligible improvements in fit when 


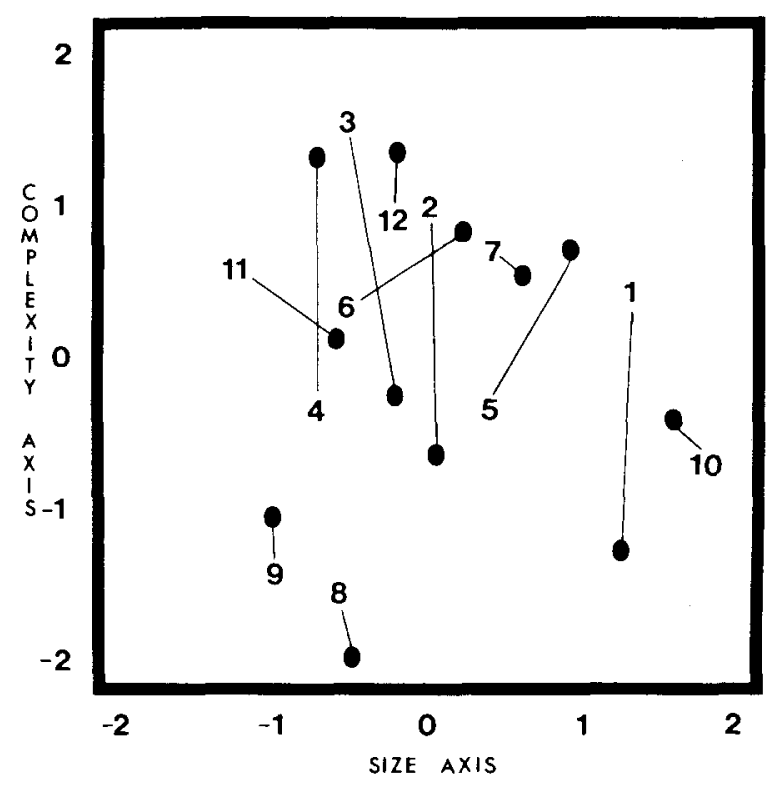

A

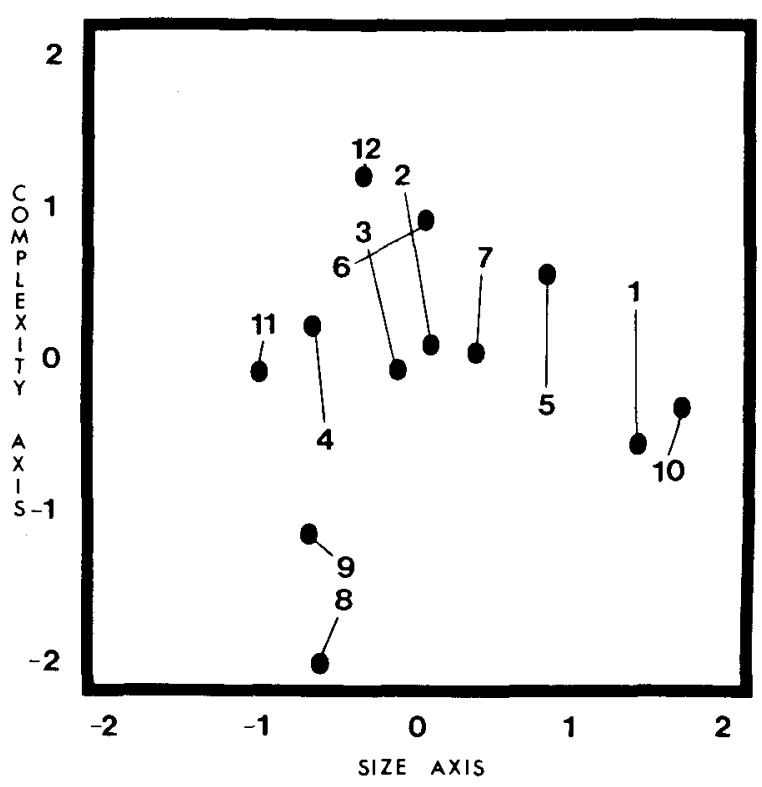

$B$

Figure 3. Two-dimensional multidimensional scaling solutions for (A) adults and (B) children, after orthonomral rotation to positions of maximum congruence and superimposition. The visual position is shown by the stimulus number; the touch position is shown by the circle. The length of the lines connecting the visual and touch stimulus positions corresponds to the cross-modal dissimilarity value of that stimulus for that age group.

a third dimension was added. The resulting twodimensional solutions for each condition are shown in Figures 2A-2D.

\section{Examination of the Reliability of the MDS Solutions}

Analyses were conducted to ensure that the data collection method had produced reliable similarity data, a necessary precursor of representative MDS solutions. This was important particularly for the child data, because more children contributed fewer data points each. The analyses were performed separately for each age $\times$ modality condition, using a split-half cross-comparison process similar to the cross-validation procedure often used to examine the stability of multiple regression models.

For each condition, one dissimilarity matrix was formed from the first set of 220 trials (representing one pass through the possible triadic combinations), and a second matrix was formed from the second set of 220 trials. A two-dimensional MDS solution was obtained from each matrix, and the pairs of solutions from each design condition were compared, using a two-step process (Cliff, 1966). First, the two MDS solutions were orthonormally rotated to positions of maximum congruence. ${ }^{3}$ Then, the coefficient of congruence (CC; which may be usefully interpreted much as a correlation coefficient) was used to assess the extent of the similarity of the two solutions for each corresponding dimension. A large $\mathrm{CC}$ value is interpreted to mean that the stimuli were similarly positioned on the corresponding dimensions from the two MDS so- lutions being compared. For example, for the child visual condition, the first dimensions of the split-half solutions had a CC value of .94 and the second dimensions had a CC value of .91 , indicating that the positions of the stimuli in the two split-half MDS solutions were similar along both the first and the second dimensions.

Using this procedure, $\mathrm{CC}$ values for the dimension-bydimension solution comparisons were .90 or larger for each of the four design conditions. These split-half comparison results provide strong evidence that the method of triads produced similarity rating data and MDS solutions from both adults and children are stable and reliable.

\section{Interpretation of Adult MDS Solutions}

The procedure described above was also used to compare these adult MDS solutions to those obtained in previ-

Table 1

$R^{2}$ and Stress Values $(S V)$ from Multidimensional Scaling Solutions

\begin{tabular}{|c|c|c|c|c|c|c|}
\hline \multirow[b]{3}{*}{ Solution } & \multicolumn{6}{|c|}{ Number of Solution Dimensions } \\
\hline & \multicolumn{2}{|c|}{1} & \multicolumn{2}{|c|}{2} & \multicolumn{2}{|c|}{3} \\
\hline & $R^{2}$ & $S V$ & $\overline{R^{2}}$ & $S V$ & $\overline{R^{2}}$ & $S V$ \\
\hline \multicolumn{7}{|c|}{ Adults } \\
\hline Visual & .74 & .20 & .93 & .12 & .94 & .11 \\
\hline Touch & .69 & .24 & .91 & .14 & .92 & .12 \\
\hline \multicolumn{7}{|c|}{ Children } \\
\hline Visual & .67 & .28 & .89 & .14 & .93 & .11 \\
\hline Touch & .68 & .26 & .87 & .15 & .89 & .12 \\
\hline
\end{tabular}


ous research (Garbin \& Bernstein, 1984). These comparisons revealed that the present adult solutions were very similar to the corresponding visual and touch solutions obtained previously. Each dimension of the present adult visual and touch solutions matched the corresponding dimension of the previous MDS model with a $\mathrm{CC}$ value larger than .93 .

One important inference that can be drawn from the strong similarity between these and the previous MDS solutions is that the dimensional descriptions obtained previously may be applied to the present adult solutions. Thus, the first, horizontal axis is related to perceived variations in size and compactness, and the second, vertical axis is related to perceived variations in the complexity and angularity of features of the stimuli. To test this inference, analyses were performed in which the attribute rating data from Garbin and Bernstein (1984) were employed. Data for attribute size, compactness, complexity, and angularity were used. Multiple linear regression analyses provide one way to interpret the dimensions of an MDS solution in terms of a given set of attributes (Kruskal \& Wish, 1978). For each analysis, the criterion variable is one of the attributes, and the predictors are the solution axes. For example, the first analysis involved three values for each of the 12 stimuli, the size rating for that stimulus was the criterion, and the positions on the horizontal and vertical MDS axes were the two predictors. For these analyses, a large $R^{2}$ indicates that the attribute vector fits into the MDS solution space. The resulting $R^{2}$ values are shown in the left side of Table 2.

Figures 2A and 2B show the positions of the four attribute vectors in the adult MDS solutions. These positions were obtained by using the inverse cosines of the normalized regression weights from each of the multiple regressions. The results are very similar to those from earlier research with these stimuli, as the MDS solutions comparisons described above would suggest, and they reveal that the similarities and differences among the shapes of these stimuli are perceived in terms of size/compactness (the dominant axis) and shape/complexity.

\section{Interpretation of Child MDS Solutions}

Analyses were conducted to allow an interpretation of the child solution axes in terms of the children's attribute perceptions. As described above, sets of stimuli were presented for children to rank according to each of four attributes: size, compactness, complexity, and angularity. For

Table 2

Multiple correlations Between Attribute Values and Multidimensional Scaling Solution Coordinates for Each Condition

\begin{tabular}{lcccccc}
\hline & \multicolumn{2}{c}{ Adults } & & \multicolumn{2}{c}{ Children } \\
\cline { 2 - 3 } \cline { 6 - 7 } \multicolumn{1}{c}{ Attribute } & Visual & Touch & & Visual & Touch \\
\hline Small versus large & .88 & .82 & & .82 & .79 \\
Compact versus dispersed & .70 & .64 & & .72 & .71 \\
Simple versus complex & .82 & .78 & & .78 & .75 \\
Angular versus round & .84 & .81 & & .88 & .80 \\
\hline
\end{tabular}

each attribute, the value of each stimulus was represented by the median ranking of that stimulus within that modality. ${ }^{4}$ The right side of Table 2 shows the results from the multiple regression analyses for the child data, and Figures 2C and 2D show the actual positions of the four attributes axes in the MDS solutions.

These results clearly indicate that, as for the adult data, the horizontal axis of both the visual and touch solutions correspond with differences in perceived size and compactness, while the vertical axis corresponds with differences in perceived complexity and angularity. Hereafter, the axes of the solutions will be referred to as the size and shape dimensions, respectively, for both age groups.

\section{Age-Group Comparison and \\ Perceptual Equivalence Analysis}

Figure 3A shows the adult visual and touch MDS solutions rotated to positions of maximum congruence and superimposed, with the visual and touch positions of each stimulus connected by a straight line. The length of this line represents the CMD value for each stimulus (hereafter called the CMD line). It should be noted that for both the superimposed adult and child solutions the six stimuli that were chosen as more equivalent have the shorter CMD lines, while the six less equivalent stimuli have the longer CMD lines. Comparison of the adult visual and touch solutions revealed $C C$ values of .91 for the size axis and .71 for the shape axis (these values were reported as .90 and .73, respectively, in the 24-stimulus analysis done by Garbin \& Bernstein, 1984). Figure 3B shows the child MDS solutions after rotation and superimposition, with the visual and touch positions of each stimulus connected by a CMD line. Comparison of the child visual and touch solutions yielded CC values of .90 for the size axis and .81 for the shape axis. As in earlier research, these dimension-by-dimension comparisons provide support for the general nature of perceptual equivalence, while highlighting the variability of this property across stimuli.

Figures $3 \mathrm{~A}$ and $3 \mathrm{~B}$ provide the information to compare the two age groups with respect to both overall perceptual structure and extent of perceptual equivalence. On first inspection, the overall structures of the two groups' solutions seem highly similar. Comparison of the adult and child visual solutions yielded CC values of .94 for the size dimension and .92 for the shape dimension, respectively. Comparison of the adult and child touch solutions yielded a CC of .93 for the size dimension, and .76 for the shape dimension. For these comparisons, the single low CC value for the shape dimension of the touch solutions, in combination with a careful inspection of the two figures, indicates a systematic difference between the touch perceptual structures of the two age groups.

It is important to note that the lower CC value for the shape dimension of the touch comparison is not produced by random differences in the positions of the stimuli in the two touch MDS solutions. Rather, the differences are highly systematic, readily interpretable, and easily 
describable in terms of the CMD lines connecting the visual and touch stimulus positions for the two age groups. Inspection of Figures $3 \mathrm{~A}$ and $3 \mathrm{~B}$ reveals that, whereas the CMD lines connecting the six more equivalent stimuli are of about the same length for the adult and child solutions, the CMD lines connecting the visual and touch positions of the six less equivalent stimuli are clearly shorter in the child solution than in the adult solution.

This interpretation is supported by the analysis described above that the visual-touch comparison of the shape axis yielded a larger $\mathrm{CC}$ value for the child solution than it did for the adult solution. Statistical analyses of the CMD values of the stimuli support this impression. A two-way (age group $\times$ stimuli-more vs. less equivalent) mixed effects ANOVA of the CMD values represented by the lines in Figures $3 \mathrm{~A}$ and $3 \mathrm{~B}$ revealed an interaction between these two variables $[F(1,10)=20.74$, $\left.p<.002, M S_{\mathrm{e}}=107.08\right]$. Simple effects analysis of the stimulus variable revealed greater differences between the more and less perceptually equivalent stimuli for adults $\left[F(1,10)=69.17, p<.01, \omega^{2}=.87\right]$ than it did for children $\left[F(1,10)=6.30, p<.05, \omega^{2}=.39\right] .{ }^{5}$ Given the evidence cited above that the child data are stable, and that the visual solutions of the two age groups are highly similar, the conclusion to be drawn from these results is that the child touch perceptual structure is identifiably different from the adult touch structure. The child visual and touch structures are more similar to each other than are those of adults, and they indicate greater perceptual equivalence than do the structures of the adult data.

\section{Summary and Conclusions}

The results can be summarized as follows: Both children and adults based their similarity judgments of these three-dimensional stimuli on attributes that can be summarized in terms of size and shape. In addition, both age groups emphasized the size attributes in their similarity judgments, as is indicated by these attributes' comprising the first MDS dimension (which accounts for the most variation in the data). The sole systematic difference among the MDS solutions of the four conditions involved the particular ordering of the stimuli along the second (complexity/angularity) dimension of the child touch solution. Comparison of this dimension with corresponding dimensions of the child visual and adult touch solutions revealed that children show greater perceptual equivalence between visual and touch solutions than do adults.

As in previous research (Garbin, 1988; Garbin \& Bernstein, 1984), the present results support the theoretical claims of Gibson (1966) and Marks (1978) regarding partial perceptual equivalence of vision and touch systems with respect to the processing of shape information. An important conclusion to be made from these data is that the MDS models of visual and touch perceptual structure for the 6-year-olds are quite similar to those obtained from the adults. In addition, the interpretations of those structures in terms of stimulus attributes are highly similar.
Differences between the child and adult solutions may be summarized by saying that the children show greater perceptual equivalence than the adults do, although the same stimuli are "less"' equivalent for both groups.

Of obvious interest is the source of the greater perceptual equivalence among the 6-year-olds. Obviously "development as integration" models will not describe the pattern of data found here. Rather, an explanation is needed that could account for the lesser "skill" of perceptual equivalence in the adults. A rather simple, yet easily testable, explanation can be proposed. Children, particularly within this age group, have a much greater tendency to touch and haptically explore shapes than adults do. Perhaps the effect found here might be best described as a practice or perhaps as a "loss of practice" effect brought about by a decline in bimodal experience with age. This explanation suggests specifically that perceptual equivalence is increased (from whatever level is initially supported by the physiological integration of the systems) by bimodal experience. This can be contrasted with explanations that cross-modal performance is limited by the performance of the "poorer" modality (cf. Jones, 1981). Tests of this explanation, now being completed, include the provision of various kinds of practice or experience to different individuals, and the attempt to determine whether or not these have any differential effect on the models of visual and/or touch perceptual structures and thus on the measures of perceptual equivalence.

The present results also draw attention to issues that relate to the theory as well as to the methodology of crossmodal performance research. Jones (1981) has suggested the importance of examining cross-modal performance by using designs that involve the factors of age, task, and important properties of the stimuli (size, complexity, etc.). The present results and those of Garbin (1988) suggest the importance of carefully selecting and examining the stimuli employed in discrimination or matching tasks, particularly in terms of their visual-touch perceptual similarity. This suggestion is a specific application of Garner's (1970) general admonition to understand the properties of stimuli used for perceptual or cognitive research. The second issue involves the importance of separating perceptual and cognitive (e.g., memory and decision bias) contributions to cross-modal performance. The not uncommon finding that the cross-modal (relative to intramodal) performances are poorer in children than in adults takes on a different and potentially more "cognitive" interpretation, given the evidence from the present data that children have somewhat greater perceptual equivalence than adults do.

\section{REFERENCES}

Abravanel, E. (1981). Integrating the information from eyes and hands: A developmental account. In R. E. Walk \& H. L. Pick, Jr. (Eds.), Intersensory perception and sensory integration (pp. 71-108). New York: Plenum.

Cliff, N. (1966). Orthogonal rotation to congruence. Psychometrika, 31, 33-42. 
GARBIN, C. P. (1988). Visual-haptic perceptual nonequivalence for shape information and its impact upon cross-modal performance. Journal of Experimental Psychology: Human Perception \& Performance, 14, 547-553.

Garbin, C. P., \& Bernstein, I. H. (1984). Visual and haptic perception of three-dimensional solid forms. Perception \& Pschophysics, 36, 104-110.

GARNER, E. R. (1970). The stimulus in information processing. American Psychologist, 25, 350-358.

GiBson, J. J. (1966). The senses considered as perceptual systems. Boston: Houghton Mifflin.

IsAAC, P. D. (1970). Dissimilarities as indices of individual perceptual structure. Perception \& Psychophysics, 7, 229-233.

JoNES, B. (1981). The developmental significance of cross-modal matching. In R. E. Walk \& H. L. Pick, Jr. (Eds.), Intersensory perception and sensory integration (pp. 109-137). New York: Plenum.

Klatzky, R. L., Lederman, S., \& Reed, C. (1987). There's more to touch than meets the eye: The salience of object attributes for haptics with and without vision. Journal of Experimental Psychology: General, 116, 356-369.

Kruskal, J. B., \& WISH, M. (1978). Multidimensional scaling. Beverly Hills, CA: Sage.

MARKS, L. E. (1978). The unity of the senses: Interrelations among the modalities. New York: Academic Press.

OWEN, D. H., \& Brown, D. R. (1970). Visual and tactual form discrimination: Psychophysical comparison within and between modalities. Perception \& Psychophysics, 7, 302-306.

Pick, A. D., Pick, H. L., \& Thomas, M. L. (1966). Cross-modal transfer and improvement of form discrimination. Journal of Experimental Child Psychology, 3, 279-288.

TORGERSON, W. S. (1958). Theory and methods of scaling. New York: Wiley.

WISH, M. (1976). Comparisons among multidimensional structures of interpersonal relations. Multivariate Behavior Research, 12, 297-324.
YounG, F. W., \& LEWYCKYJ, R. (1979). ALSCAL-4: User's guide. University of North Carolina, Chapel Hill: Department of Psychology

\section{NOTES}

1. It should be noted, however, that this line of reasoning ignores interpretations of poorer cross-modal performance in children that focus on the "motor" and "visual guidance" aspects of touch and crossmodal performance (e.g., Jones, 1981).

2 . For the adults, a symmetry attribute was collected that involved a rating of the "overall" symmetry, as opposed to the symmetry of the stimulus perceived from a particular orientation. Attempts to collect these data from pilot children produced very low interrater reliabilities.

3. These and all subsequent MDS solution comparison analyses were also performed using a Procrustes-type algorithm, which adjusted the relative length of the axes as well as their relative position to ensure maximum congruence of the solutions. As noted in Garbin (1988), these two procedures usually produce very similar results when the variance accounted for by the two models (and so the range along the axes) is similar, as they did in the present analyses.

4. This procedure was adopted to simplify the presentation of the analysis. Parallel analyses in which nonparametric procedures were employed produced the same statistical and substantive conclusions.

5. In addition, there were main effects of age $[F(1,10)=29.45$, $p<.01]$ and stimulus $[F(1,10)=63.54, p<.01]$. The same pattern of statistical results was found when the CMD values from the MDS solutions of the first and second set of 220 trials were analyzed in separate $2 \times 2$ ANOVAS.

\title{
Notices and Announcements
}

\author{
6th European Conference on Eye Movements \\ Leuven, Belgium \\ September 15-18, 1991
}

Call for Papers

Deadline: January 31, 1991

(Manuscript received November 2, 1988; accepted for publication March 20, 1990.)

The 6 th European Conference on Eye Movements will be held at the University of Leuven, September 15-18, 1991. Its aim is to promote the wider exchange of information concerning eye-movement research in all its diverse fields, and to encourage contact beween basic and applied research. The Conference will be of interest to psychologists, educational scientists, neurophysiologists, medical doctors, bioengineers, ergonomists, and others interested in visual science.

Papers and posters on the following topics are welcome: neurophysiology of eye movement, oculomotor system, measurement techiques, eye movements in perceptual and cognitive tasks, eye movements and reading, oculomotor disorders, and applied research. Papers integrating sensory sciences and higher order studies will be favored. Papers and posters should be in English, and paper presentations should not exceed 20 minutes.

One-page abstracts for spoken and poster presentations should be sent, by January 31, 1991, to: Laboratorium voor Experimentele Psychologie, Katholieke Universiteit Leuven, Tiensestraat 102, B-3000 Leuven, Belgium (e-mail: fpaas91@blekul11 earn (bitnet)).

Further information may be obtained from Géry d'Ydewalle or Johan Van Rensbergen at the address given above. 